\title{
POSITIVE TOEPLITZ OPERATORS FROM A HARMONIC BERGMAN SPACE INTO ANOTHER
}

\author{
Boo R. Choe, Young J. Lee And Kyunguk NA
}

(Received September 30, 2002, revised August 4, 2003)

\begin{abstract}
On the setting of bounded smooth domains, we study positive Toeplitz operators between the harmonic Bergman spaces. We give characterizations of bounded and compact Toeplitz operators taking one harmonic Bergman space into another in terms of certain Carleson and vanishing Carleson measures.
\end{abstract}

1. Introduction. Throughout this paper, we let $\Omega$ be a fixed smooth bounded domain in $\boldsymbol{R}^{n}$ for $n \geq 2$. For $1 \leq p<\infty$, the harmonic Bergman space $b^{p}$ is the set of all complexvalued harmonic functions $f$ on $\Omega$ such that

$$
\|f\|_{p}=\left\{\int_{\Omega}|f|^{p} d V\right\}^{1 / p}<\infty,
$$

where $V$ denotes the Lebesgue volume measure on $\Omega$. We will also use the notation $d y=$ $d V(y)$ for simplicity. Also, $b^{\infty}$ denotes the space of all bounded harmonic functions on $\Omega$. It is known that $b^{\infty}$ is dense in each $b^{p}$.

As is well-known, the space $b^{p}$ is a closed subspace of $L^{p}=L^{p}(\Omega, V)$ and hence a Banach space. In particular, $b^{2}$ is a Hilbert space. Each point evaluation is easily verified to be a bounded linear functional on $b^{2}$. Hence, for each $x \in \Omega$, there exists a unique function $R(x, \cdot) \in b^{2}$ which has the following reproducing property:

$$
f(x)=\int_{\Omega} f(y) \overline{R(x, y)} d y, \quad x \in \Omega
$$

for all $f \in b^{2}$.

Let $Q$ be the Hilbert space orthogonal projection from $L^{2}$ onto $b^{2}$. The reproducing kernels $R(x, \cdot)$ are known to be symmetric and real-valued. Hence, the reproducing property mentioned above yields the following integral representation of $Q$ :

$$
Q \psi(x)=\int_{\Omega} \psi(y) R(x, y) d y, \quad x \in \Omega
$$

for functions $\psi \in L^{2}$. See [2] for more information and related facts.

For each fixed $x \in \Omega$, the function $R(x, \cdot)$ is known to be bounded on $\Omega$. Thus, the projection $Q$ naturally extends to an integral operator via (1.1) from $L^{1}$ into the space of all

2000 Mathematics Subject Classification. Primary 47B35; Secondary 31B05.

Key words and phrases. Toeplitz operators, Harmonic Bergman spaces, Carleson measure.

This work was supported by KOSEF (R01-1998-000-00119-0) and Korea University Grant (2002). 
harmonic functions on $\Omega$. Moreover, for $1<p<\infty$, it is known that $Q$ is a bounded projection from $L^{p}$ onto $b^{p}$. The integral transform $Q$ even extends to $M(\Omega)$, the space of all complex Borel measures on $\Omega$. Namely, for each $\mu \in M(\Omega)$, the integral

$$
Q \mu(x)=\int_{\Omega} R(x, y) d \mu(y), \quad x \in \Omega
$$

defines a function harmonic on $\Omega$. See [4] or [6] for details.

For $\mu \in M(\Omega)$, the Toeplitz operator $T_{\mu}$ with symbol $\mu$ is defined by

$$
T_{\mu} f=Q(f d \mu)
$$

for $f \in b^{\infty}$. In case $\mu=\varphi d V$, we will write $T_{\mu}=T_{\varphi}$. Note that $T_{\mu}$ is densely defined on $b^{p}$ for each $1<p<\infty$.

Toeplitz operators acting on holomorphic Bergman spaces have been well studied. Especially, positive symbols of bounded and compact Toeplitz operators are completely characterized in terms of Carleson type measures as in [9], [13] and references therein. Recently, the analogous characterizations for harmonic Bergman spaces have been obtained in [10], [11] on the ball and then in [4] on smooth bounded domains; these results are all concerned with Toeplitz operators from a Bergman space into itself.

In a recent paper [3] where the setting is the half-space, Toeplitz operators from a Bergman space into another are considered and positive symbols of bounded and compact Toeplitz operators are characterized. In this paper, we prove analogous results on general smooth bounded domains. Our results show that known results as in [3] continue to hold in this general setting.

In Section 2, we collect some preliminary results related to certain averaging functions and Berezin transforms. In Section 3, we characterize Carleson measures in terms of the averaging functions and Berezin transforms. These results will be used to prove our characterizations of Toeplitz operators in Section 4.

2. Averaging functions and Berezin transforms. Throughout this paper, we will often abbreviate inessential constants involved in inequalities by writing $A \lesssim B$ for positive quantities $A$ and $B$ if the ratio $A / B$ has a positive upper bound. Also, we write $A \approx B$ if $A \lesssim B$ and $B \lesssim A$.

For $x \in \Omega$, we let $r(x)=\operatorname{dist}(x, \partial \Omega)$ and

$$
E_{\delta}(x)=\{y \in \Omega ;|x-y|<\delta r(x)\}
$$

for $0<\delta<1$. One can easily see that

$$
(1-\delta) r(x)<r(y)<(1+\delta) r(x)
$$

for $x \in \Omega$ and $y \in E_{\delta}(x)$. Since $\delta<1$, we note that $E_{\delta}(x)$ is the euclidean ball with center at $x$ and radius $\delta r(x)$. So, we have $V\left(E_{\delta}(x)\right) \approx r(x)^{n}$ for $x \in \Omega$. 
Let $\delta \in(0,1)$. Given $\mu \in M(\Omega)$, the averaging function $\hat{\mu}_{\delta}$ of $\mu$ over the balls $E_{\delta}(x)$ is defined by

$$
\hat{\mu}_{\delta}(x)=\frac{\mu\left(E_{\delta}(x)\right)}{V\left(E_{\delta}(x)\right)}, \quad x \in \Omega .
$$

Also, for $1<t<\infty$, we define the Berezin $t$-transform $\tilde{\mu}_{t}$ on $\Omega$ by

$$
\tilde{\mu}_{t}(x)=\int_{\Omega}\left|k_{x, t}(y)\right|^{t} d \mu(y), \quad x \in \Omega,
$$

where

$$
k_{x, t}(y)=\frac{R(x, y)}{\|R(x, \cdot)\|_{t}}, \quad y \in \Omega
$$

is the $L^{t}$-normalized reproducing kernel. In case $d \mu=f d V$ for $f \in L^{1}$, we will write $\hat{\mu}_{\delta}=\hat{f}_{\delta}$ and $\tilde{\mu}_{t}=\tilde{f}_{t}$ for simplicity.

We start with the $L^{p}$-boundedness of the averaging operator.

PROPOSITION 2.1. The averaging operator $f \mapsto \hat{f}_{\delta}$ is bounded on $L^{p}$ for each $1 \leq$ $p \leq \infty$ and $0<\delta<1$.

Proof. Clearly, the averaging operator is bounded on $L^{\infty}$. So, we now assume $1 \leq$ $p<\infty$ and let $0<\delta<1$. For $y \in E_{\delta}(x)$ we note $x \in D(y)$ by (2.1), where

$$
D(y)=\left\{x \in \boldsymbol{R}^{n} ;|x-y|<\delta r(y) /(1-\delta)\right\} .
$$

Thus, for $f \in L^{p}$, we have by Jensen's inequality and (2.1) that

$$
\begin{aligned}
\int_{\Omega}\left|\hat{f}_{\delta}(x)\right|^{p} d x & \leq \int_{\Omega}\left\{\frac{1}{V\left(E_{\delta}(x)\right)} \int_{E_{\delta}(x)}|f(y)| d y\right\}^{p} d x \\
& \lesssim \int_{\Omega} \frac{1}{r(x)^{n}} \int_{E_{\delta}(x)}|f(y)|^{p} d y d x \\
& \approx \int_{\Omega} \int_{E_{\delta}(x)} \frac{1}{r(y)^{n}}|f(y)|^{p} d y d x \\
& \leq \int_{\Omega}|f(y)|^{p} \frac{1}{r(y)^{n}} \int_{D(y)} 1 d x d y \\
& \approx \int_{\Omega}|f(y)|^{p} d y .
\end{aligned}
$$

We also need the following submean-value type inequality for averaging functions of positive finite Borel measures $\mu$ on $\Omega$ (we simply write $\mu \geq 0$ ).

Lemma 2.2. Let $\delta, \varepsilon \in(0,1)$. Then there exist constants $C_{\delta, \varepsilon}$ such that

$$
\hat{\mu}_{\delta}(x) \leq \frac{C_{\delta, \varepsilon}}{V\left(E_{\delta}(x)\right)} \int_{E_{\delta}(x)} \hat{\mu}_{\varepsilon} d V
$$

for all $\mu \geq 0$ and $x \in \Omega$.

Proof. See Lemma 3.2 of [4]. 
Combining the above with Proposition 2.1 , we see that $L^{p}$-behavior of $\hat{\mu}_{\delta}$ of a given measure $\mu \geq 0$ is independent of $\delta$.

PROPOSITION 2.3. Let $1 \leq p \leq \infty$ and $\mu \geq 0$. If $\hat{\mu}_{\varepsilon} \in L^{p}$ for some $\varepsilon \in(0,1)$, then $\hat{\mu}_{\delta} \in L^{p}$ for all $\delta \in(0,1)$.

Proof. By Lemma 2.2, we have $\left.\hat{\mu}_{\delta} \lesssim \widehat{\left[\hat{\mu}_{\varepsilon}\right.}\right]_{\delta}$ for each fixed $\delta, \varepsilon \in(0,1)$. Thus, the result follows from Proposition 2.1.

Given $\delta \in(0,1)$ and a sequence $\left\{x_{m}\right\}$ in $\Omega$, we say that $\left\{x_{m}\right\}$ is $\delta$-separated if the sets $E_{\delta}\left(x_{m}\right)$ are pairwise disjoint. Next, we need the following covering lemma whose proof is essentially the same as that in [7] or [12]. So, we omit the details.

LEMMA 2.4. Let $\delta \in(0,1)$. Then there exists a sequence $\left\{a_{m}\right\}$ in $\Omega$ satisfying the following conditions:

(a) $\left\{a_{m}\right\}$ is an $\delta / 6$-separated sequence.

(b) $\bigcup_{m} E_{\delta / 3}\left(a_{m}\right)=\Omega$.

(c) There is a positive integer $N=N(n, \delta)$ such that each point in $\Omega$ belongs to at most $N$ of the balls $E_{\delta}\left(a_{m}\right)$.

Note that $a_{m} \rightarrow \partial \Omega$ as $m \rightarrow \infty$. Whenever we use expressions like $\hat{\mu}_{\delta}\left(a_{m}\right)$ in what follows, the sequence $\left\{a_{m}\right\}=\left\{a_{m}(\delta)\right\}$ will always refer to the sequence chosen in Lemma 2.4 .

Proposition 2.5. Let $1 \leq p<\infty$ and $\varepsilon, \delta \in(0,1)$. Then, for any $\mu \geq 0$, we have $\hat{\mu}_{\varepsilon} \in L^{p}$ if and only if $\sum_{m}\left|\hat{\mu}_{\delta}\left(a_{m}\right)\right|^{p} r\left(a_{m}\right)^{n}<\infty$.

Proof. First, assume $\hat{\mu}_{\varepsilon} \in L^{p}$. By Lemma 2.2 and Jensen's inequality, we have

$$
\sum_{m}\left|\hat{\mu}_{\delta}\left(a_{m}\right)\right|^{p} r\left(a_{m}\right)^{n} \lesssim \sum_{m} \int_{E_{\delta}\left(a_{m}\right)}\left|\hat{\mu}_{\varepsilon}\right|^{p} d V \leq N \int_{\Omega}\left|\hat{\mu}_{\varepsilon}\right|^{p} d V<\infty,
$$

where $N$ is the positive integer provided by Lemma 2.4.

Conversely, suppose $\sum_{m}\left|\hat{\mu}_{\delta}\left(a_{m}\right)\right|^{p} r\left(a_{m}\right)^{n}<\infty$. For $a \in \Omega$ and $x \in E_{\delta / 3}(a)$, we note that $E_{\delta / 3}(x) \subset E_{\delta}(a)$. It follows from (2.1) that

$$
\hat{\mu}_{\delta / 3}(x) \leq \hat{\mu}_{\delta}\left(a_{m}\right) \frac{V\left(E_{\delta}\left(a_{m}\right)\right)}{V\left(E_{\delta / 3}(x)\right)} \lesssim \hat{\mu}_{\delta}\left(a_{m}\right), \quad x \in E_{\delta / 3}\left(a_{m}\right)
$$

for $m=1,2, \ldots$. Thus, we have

$$
\begin{aligned}
\int_{\Omega}\left|\hat{\mu}_{\delta / 3}\right|^{p} d V & \leq \sum_{m} \int_{E_{\delta / 3}\left(a_{m}\right)}\left|\hat{\mu}_{\delta / 3}(x)\right|^{p} d V(x) \\
& \lesssim \sum_{m}\left|\hat{\mu}_{\delta}\left(a_{m}\right)\right|^{p} V\left(E_{\delta / 3}\left(a_{m}\right)\right) \\
& \approx \sum_{m}\left|\hat{\mu}_{\delta}\left(a_{m}\right)\right|^{p} r\left(a_{m}\right)^{n}
\end{aligned}
$$

So, we have $\hat{\mu}_{\delta / 3} \in L^{p}$. Now, by Proposition 2.3, we have $\hat{\mu}_{\varepsilon} \in L^{p}$. 
Next, we prove the $L^{p}$-boundedness of Berezin transforms. Before doing this, we first need a couple of lemmas. In what follows, given $1<p<\infty, p^{\prime}$ always denotes the conjugate exponent of $p$. Also, we let $d(x, y)=|x-y|+r(x)+r(y)$ for $x, y \in \Omega$.

LEMMA 2.6. There exists a constant $C_{n}$ such that

$$
|R(x, y)| \leq \frac{C_{n}}{d(x, y)^{n}}
$$

for $x, y \in \Omega$.

PROOF. See Theorem 1.1 of [6].

Lemma 2.7. Let $1<p<\infty$. Then there is a constant $C_{p}$ such that

$$
C_{p}^{-1} \leq\|R(x, \cdot)\|_{p} r(x)^{n / p^{\prime}} \leq C_{p}
$$

for every $x \in \Omega$.

Proof. See Lemma 2.4 of [4].

Lemma 2.8. For $s, t \geq 0$ with $s+t>0$ and $t<1$, there exists a constant $C_{s, t}$ such that

for $x \in \Omega$.

$$
\int_{\Omega} \frac{d y}{d(x, y)^{n+s} r(y)^{t}} \leq \frac{C_{s, t}}{r(x)^{s+t}}
$$

Proof. See Lemma 4.1 of [6].

For $f \in L^{1}$, we define

$$
\Phi f(x)=\int_{\Omega} \frac{f(y)}{d(x, y)^{n}} d y, \quad x \in \Omega .
$$

LEMMA 2.9. For $1<p<\infty, \Phi: L^{p} \rightarrow L^{p}$ is bounded.

PROOF. This is implicit in the proof of Theorem 4.2 on [6].

Proposition 2.10. Let $1<p \leq \infty$ and $1<t<\infty$. Then the Berezin $t$-transform $f \mapsto \tilde{f}_{t}$ is bounded on $L^{p}$.

Proof. The case $p=\infty$ is clear. Now, let $f \in L^{1}$. Since $r(x) \leq d(x, y)$ for all $x, y \in \Omega$, we have by Lemma 2.6 and Lemma 2.7

$$
\left|\tilde{f}_{t}(x)\right| \lesssim r(x)^{t n-n} \int_{\Omega} \frac{|f(y)|}{d(x, y)^{t n}} d y \leq \int_{\Omega} \frac{|f(y)|}{d(x, y)^{n}} d y=\Phi|f|(x), \quad x \in \Omega .
$$

Thus, the $L^{p}$-boundedness of the Berezin $t$-transform is a consequence of Lemma 2.9.

We now turn to relations between $L^{p}$-behavior of averaging functions and Berezin transforms. We first prove the following.

LEMMA 2.11. Given $\delta \in(0,1)$, there is a constant $C_{\delta}$ such that

$$
\int_{\Omega} f d \mu \leq C_{\delta} \int_{\Omega} f \hat{\mu}_{\delta} d V
$$


for all $f \geq 0$ subharmonic on $\Omega$ and $\mu \geq 0$.

ProOF. Fix $\delta \in(0,1)$. Let $\mu \geq 0$ and $f$ be a positive subharmonic function. Note that if $y \in E_{\delta /(1+\delta)}(x)$, then $x \in E_{\delta}(y)$. In other words, we have

$$
\chi_{E_{\delta /(1+\delta)}(x)}(y) \leq \chi_{E_{\delta}(y)}(x)
$$

for $x, y \in \Omega$. Also, since $r(x) \approx r(y)$ for $y \in E_{\delta}(x)$, we note

$$
\int_{E_{\delta}(y)} \frac{1}{V\left(E_{\delta /(1+\delta)}(x)\right)} d \mu(x) \approx \int_{E_{\delta}(y)} \frac{1}{r(x)^{n}} d \mu(x) \approx \frac{\mu\left(E_{\delta}(y)\right)}{r(y)^{n}} \approx \hat{\mu}_{\delta}(y)
$$

for $y \in \Omega$. Thus, it follows from subharmonicity and Fubini's theorem that

$$
\begin{aligned}
\int_{\Omega} f(x) d \mu(x) & \leq \int_{\Omega} \frac{1}{V\left(E_{\delta /(1+\delta)}(x)\right)} \int_{E_{\delta /(1+\delta)}(x)} f(y) d y d \mu(x) \\
& \leq \int_{\Omega} \int_{\Omega} f(y) \frac{1}{V\left(E_{\delta /(1+\delta)}(x)\right)} \chi_{E_{\delta}(y)}(x) d y d \mu(x) \\
& =\int_{\Omega} f(y) \int_{E_{\delta}(y)} \frac{1}{V\left(E_{\delta /(1+\delta)}(x)\right)} d \mu(x) d y \\
& \approx \int_{\Omega} f \hat{\mu}_{\delta} d V .
\end{aligned}
$$

LEMMA 2.12. There exists a $\delta_{0} \in(0,1)$ such that $R(x, y) \approx r(x)^{-n}$ whenever $x \in \Omega$ and $y \in E_{\delta_{0}}(x)$.

Proof. See Lemma 2.3 of [4].

In what follows, $\delta_{0}$ will always denote the number provided in Lemma 2.12.

LEMMA 2.13. Given $0<\delta<\delta_{0}$ and $1<t<\infty$, there exists a constant $C=C_{\delta, t}$ such that $\hat{\mu}_{\delta} \leq C \tilde{\mu}_{t}$ for any $\mu \geq 0$.

PROOF. Let $x \in \Omega$. Then, by Lemma 2.7 and Lemma 2.12, we have

$$
\int_{E_{\delta_{0}}(x)}\left|k_{x, t}\right|^{t} d \mu \approx \frac{\mu\left(E_{\delta_{0}}(x)\right)}{r(x)^{n}} \approx \hat{\mu}_{\delta_{0}}(x)
$$

so that

$$
\hat{\mu}_{\delta}(x) \lesssim \hat{\mu}_{\delta_{0}}(x) \lesssim \int_{\Omega}\left|k_{x, t}\right|^{t} d \mu=\tilde{\mu}_{t}(x)
$$

Proposition 2.14. Let $\delta \in(0,1)$ and $1<t<\infty$. Suppose $\mu \geq 0$ and $1<p \leq \infty$. Then, $\hat{\mu}_{\delta} \in L^{p}$ if and only if $\tilde{\mu}_{t} \in L^{p}$.

Proof. First, suppose $\hat{\mu}_{\delta} \in L^{p}$. Applying Lemma 2.11 to functions $f=\left|k_{x, t}\right|^{t}$, we obtain $\tilde{\mu}_{t} \lesssim \widetilde{\left[\hat{\mu}_{\delta}\right]_{t}}$ and thus $\tilde{\mu}_{t} \in L^{p}$ by Proposition 2.10. Conversely, if $\tilde{\mu}_{t} \in L^{p}$, then by Lemma 2.13, we have $\hat{\mu}_{\delta} \in L^{p}$ for $\delta<\delta_{0}$. But, Proposition 2.3 yields $\hat{\mu}_{\delta} \in L^{p}$ for a given $\delta$. 
3. Carleson measures. To characterize Toeplitz operators, we need the notion of certain Carleson measures. Let $1<p, q<\infty$. Given $\mu \geq 0$, we say that $\mu$ is a $(p, q)$-Carleson measure if there exists a constant $C$ such that

$$
\left\{\int_{\Omega}|f|^{q} d \mu\right\}^{1 / q} \leq C\|f\|_{p}
$$

for all $f \in b^{p}$. In other words, $\mu$ is a $(p, q)$-Carleson measure if and only if the inclusion $i_{p, q}: b^{p} \rightarrow L^{q}(\mu)$ is bounded. Carleson measures on various settings have been well studied as in [1], [5], [8], [11], [13] and references therein.

In this section, we also characterize $(p, q)$-Carleson measures in terms of $L^{p}$-behavior of the averaging functions and Berezin transforms. We first consider the case where $p \leq q$. The special case of $p=q$ was proved in [4].

TheOrem 3.1. Assume $1<p \leq q<\infty, s=p / q, 1 / s<t<\infty$ and $\varepsilon, \delta \in(0,1)$. Suppose $\mu \geq 0$. Then the following conditions are all equivalent:

(a) $\mu$ is a $(p, q)$-Carleson measure.

(b) $\sup _{x \in \Omega} \tilde{\mu}_{t}(x) r(x)^{n(1-1 / s)}<\infty$.

(c) $\sup _{x \in \Omega} \hat{\mu}_{\varepsilon}(x) r(x)^{n(1-1 / s)}<\infty$.

(d) $\sup _{m} \hat{\mu}_{\delta}\left(a_{m}\right) r\left(a_{m}\right)^{n(1-1 / s)}<\infty$.

Proof. First, suppose (a) and show (b) with $t=q$. Let $x \in \Omega$. By Lemma 2.7 we have

$$
\left|k_{x, p}\right|^{q}=\left(\frac{\|R(x, \cdot)\|_{q}}{\|R(x, \cdot)\|_{p}}\right)^{q}\left|k_{x, q}\right|^{q} \approx r(x)^{n(1-q / p)}\left|k_{x, q}\right|^{q} .
$$

Integrating with respect to $d \mu$, we obtain

$$
\tilde{\mu}_{q}(x) r(x)^{n(1-q / p)} \approx \int_{\Omega}\left|k_{x, p}\right|^{q} d \mu .
$$

Since $\left\|k_{x, p}\right\|_{p}=1$ and $\mu$ is a $(p, q)$-Carleson measure, the above shows that (b) holds for $t=q$.

Next, suppose (b) and show (c). By Lemma 2.13, we have (c) for $\varepsilon<\delta_{0}$. Thus, by an simple application of Lemma 2.2, we see that (c) also holds for a given $\varepsilon$.

The implication $(c) \Rightarrow(d)$ can also be easily seen from Lemma 2.2 again and (2.1).

Now, suppose (d) and show (a). Let $f \in b^{p}$. The proof of Theorem 3.5 of [4] shows that there is a constant $C=C_{\delta}$ such that

$$
\sup _{x \in E_{\delta / 3}(a)}|f(x)|^{p} \leq \frac{C}{r(a)^{n}} \int_{E_{\delta}(a)}|f|^{p} d V
$$

for all $a \in \Omega$. Hence, we have

$$
\begin{aligned}
\int_{E_{\delta / 3}(a)}|f|^{q} d \mu & \lesssim \frac{\mu\left(E_{\delta}(a)\right)}{r(a)^{q n / p}}\left\{\int_{E_{\delta}(a)}|f|^{p} d V\right\}^{q / p} \\
& \approx \hat{\mu}_{\delta}(a) r(a)^{n(1-q / p)}\left\{\int_{E_{\delta}(a)}|f|^{p} d V\right\}^{q / p}
\end{aligned}
$$


for $a \in \Omega$. Note that $q / p \geq 1$. Let $M=\sup _{m} \hat{\mu}_{\delta}\left(a_{m}\right) r\left(a_{m}\right)^{n(1-1 / s)}$. It follows from Lemma 2.4 that

$$
\begin{aligned}
\int_{\Omega}|f|^{q} d \mu & \leq \sum \int_{E_{\delta / 3}\left(a_{m}\right)}|f|^{q} d \mu \\
& \lesssim \sum \hat{\mu}_{\delta}\left(a_{m}\right) r\left(a_{m}\right)^{n(1-1 / s)}\left\{\int_{E_{\delta}\left(a_{m}\right)}|f|^{p} d V\right\}^{q / p} \\
& \leq M \sum\left\{\int_{E_{\delta}\left(a_{m}\right)}|f|^{p} d V\right\}^{q / p} \\
& \leq M\left\{\sum \int_{E_{\delta}\left(a_{m}\right)}|f|^{p} d V\right\}^{q / p} \\
& \leq N^{q / p} M\|f\|_{p}^{q},
\end{aligned}
$$

where $N$ is the number provided by Lemma 2.4. Hence, $\mu$ is a $(p, q)$-Carleson measure, as desired.

Finally, suppose (a) and show (b) for general $t$. We have seen that Condition (a) is equivalent to Condition (c), which does not depend on particular values of $p$ and $q$, but depends on the ratio $s=p / q$. Therefore, we may take $p=s t>1$ and $q=t$ in order to see that (a) implies (b) for general $t$.

Let $1<p<\infty$ and $\left\{x_{m}\right\}$ be a sequence in $\Omega$. For a sequence $\lambda=\left\{\lambda_{m}\right\} \in \ell^{p}$, we let $S(\lambda)$ be the function defined by

$$
S(\lambda)(x)=\sum \lambda_{m} r\left(x_{m}\right)^{n(1-1 / p)} R\left(x, x_{m}\right), \quad x \in \Omega .
$$

Proposition 3.2. Let $1<p<\infty$. Then $S: \ell^{p} \rightarrow b^{p}$ is bounded whenever $\left\{x_{m}\right\}$ is $\delta$-separated for some $\delta$.

Proof. By Lemma 2.6, we see

$$
\left|R\left(x, x_{m}\right)\right| \lesssim \frac{1}{d\left(x, x_{m}\right)^{n}} \lesssim \frac{1}{d(x, y)^{n}}
$$

for all $y \in E_{\delta}\left(x_{m}\right)$ and $x \in \Omega$. It follows that

$$
|S(\lambda)(x)| \lesssim \sum\left|\lambda_{m}\right| r\left(x_{m}\right)^{n(1-1 / p)} V\left(E_{\delta}\left(x_{m}\right)\right)^{-1} \int_{E_{\delta}\left(x_{m}\right)} \frac{1}{d(x, y)^{n}} d y
$$

for $x \in \Omega$. Thus, setting

$$
f=\sum\left|\lambda_{m}\right| r\left(x_{m}\right)^{n(1-1 / p)} V\left(E_{\delta}\left(x_{m}\right)\right)^{-1} \chi_{E_{\delta}\left(x_{m}\right)},
$$


we have $|S(\lambda)| \lesssim \Phi f$, where $\Phi$ is the operator defined in (2.3). Now, since $\left\{x_{m}\right\}$ is $\delta$ separated, we have by Lemma 2.9 ,

$$
\begin{aligned}
\|S(\lambda)\|_{p}^{p} & \lesssim\|f\|_{p}^{p} \\
& =\sum\left|\lambda_{m}\right|^{p} r\left(x_{m}\right)^{n(p-1)} V\left(E_{\delta}\left(x_{m}\right)\right)^{1-p} \\
& \approx \sum\left|\lambda_{m}\right|^{p},
\end{aligned}
$$

which shows that $S: \ell^{p} \rightarrow L^{p}$ is bounded and the series in (3.2) converges in norm. Since each term is harmonic, the series converges uniformly on every compact subsets of $\Omega$. It follows that $Q$ maps $\ell^{p}$ into $b^{p}$.

In order to characterize $(p, q)$-Carleson measures for $q<p$, we will utilize Luecking's idea in [7]. To do so, we first need Khinchine's inequality. Recall that the Rademacher functions $\psi_{m}$ are defined by

$$
\psi_{0}(t)=\left\{\begin{aligned}
1 & \text { if } 0 \leq t-[t]<1 / 2 \\
-1 & \text { if } 1 / 2 \leq t-[t]<1
\end{aligned}\right.
$$

and $\psi_{m}(t)=\psi_{0}\left(2^{m} t\right)$ for positive integers $m$. Then Khinchine's inequality is the following.

LEMMA 3.3 (Khinchine's inequality). For $0<p<\infty$, there exists a constant $C_{p}$ such that

$$
C_{p}^{-1}\left(\sum_{k=1}^{m}\left|\lambda_{k}\right|^{2}\right)^{p / 2} \leq \int_{0}^{1}\left|\sum_{k=1}^{m} \lambda_{k} \psi_{k}(t)\right|^{p} d t \leq C_{p}\left(\sum_{k=1}^{m}\left|\lambda_{k}\right|^{2}\right)^{p / 2}
$$

for all $m \geq 1$ and complex numbers $\lambda_{1}, \lambda_{2}, \ldots, \lambda_{m}$.

We now characterize $(p, q)$-Carleson measures for the case $q<p$.

THEOREM 3.4. Let $1<q<p<\infty, s=p / q, 1<t<\infty$ and $\delta, \varepsilon \in(0,1)$. Suppose $\mu \geq 0$. Then the following conditions are equivalent:

(a) $\mu$ is a $(p, q)$-Carleson measure.

(b) $\sum_{m}\left|\hat{\mu}_{\delta}\left(a_{m}\right)\right|^{s^{\prime}} r\left(a_{m}\right)^{n}<\infty$.

(c) $\hat{\mu}_{\varepsilon} \in L^{s^{\prime}}$.

(d) $\tilde{\mu}_{t} \in L^{s^{\prime}}$.

Proof. Assume (a) and show (b). First, consider $\delta=\delta_{0}$. Corresponding to each $\left\{\lambda_{m}\right\} \in \ell^{p}$, we put

$$
f=\sum \lambda_{m} r\left(a_{m}\right)^{n(1-1 / p)} R\left(\cdot, a_{m}\right) .
$$

Since $\left\{a_{m}\right\}$ is $\delta / 6$-separated, by Lemma 3.2, we have $\|f\|_{p} \lesssim\left\|\left(\lambda_{m}\right)\right\|_{\ell p}$ and hence, by Assumption (a),

$$
\int_{\Omega}\left|\sum \lambda_{m} r\left(a_{m}\right)^{n(1-1 / p)} R\left(x, a_{m}\right)\right|^{q} d \mu(x) \lesssim\left(\sum\left|\lambda_{m}\right|^{p}\right)^{q / p} .
$$

In the above inequality, replace $\lambda_{m}$ with $\psi_{m}(t) \lambda_{m}$ and then integrate with respect to $t$ from 0 to 1 . Then, after making use of Fubini's theorem and Khinchine's inequality, the result 
becomes

$$
\int_{\Omega}\left(\sum\left|\lambda_{m}\right|^{2} r\left(a_{m}\right)^{2 n(1-1 / p)}\left|R\left(x, a_{m}\right)\right|^{2}\right)^{q / 2} d \mu(x) \lesssim\left(\sum\left|\lambda_{m}\right|^{p}\right)^{q / p} .
$$

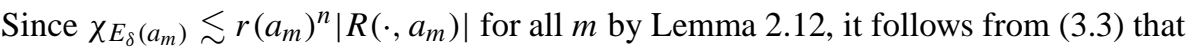

$$
\begin{aligned}
\sum\left|\lambda_{m}\right|^{q} & \hat{\mu}_{\delta}\left(a_{m}\right) r\left(a_{m}\right)^{n(1-q / p)} \\
& =\int_{\Omega} \sum\left|\lambda_{m}\right|^{q} r\left(a_{m}\right)^{-n q / p} \chi_{E_{\delta}\left(a_{m}\right)} d \mu \\
& \lesssim \max \left\{N^{1-q / 2}, 1\right\} \int_{\Omega}\left(\sum\left|\lambda_{m}\right|^{2} \chi_{E_{\delta}\left(a_{m}\right)} r\left(a_{m}\right)^{-2 n / p}\right)^{q / 2} d \mu \\
& \lesssim \int_{\Omega}\left(\sum\left|\lambda_{m}\right|^{2} r\left(a_{m}\right)^{2 n(1-1 / p)}\left|R\left(x, a_{m}\right)\right|^{2}\right)^{q / 2} d \mu \\
& \lesssim\left(\sum\left|\lambda_{m}\right|^{p}\right)^{q / p},
\end{aligned}
$$

where $N$ is the number provided in Lemma 2.4. This shows that

$$
\sum\left|b_{m}\right| \hat{\mu}_{\delta}\left(a_{m}\right) r\left(a_{m}\right)^{n / s^{\prime}} \lesssim\left(\sum\left|b_{m}\right|^{s}\right)^{1 / s}
$$

for all $\left\{b_{m}\right\} \in \ell^{s}$. Thus, a duality argument yields (b) for $\delta=\delta_{0}$. Now, an application of Proposition 2.5 shows that (b) holds for a given $\delta$.

The implication (b) $\Rightarrow$ (c) is also a consequence of Proposition 2.5.

Now, assume (c) and show (a). Using Lemma 2.11 and Hölder's inequality, we have

$$
\int_{\Omega}|f|^{q} d \mu \lesssim \int_{\Omega}|f|^{q} \hat{\mu}_{\varepsilon} d V \leq\|f\|_{p}^{q}\left\|\hat{\mu}_{\varepsilon}\right\|_{s^{\prime}}
$$

for $f \in b^{p}$ so that (a) holds.

Finally, the equivalence (c) $\Leftrightarrow$ (d) is a consequence of Proposition 2.14.

For $\mu \geq 0$ and $1<p, q<\infty$, we say that $\mu$ is a vanishing $(p, q)$-Carleson measure if the inclusion $i_{p, q}: b^{p} \rightarrow L^{q}(\mu)$ is compact, or equivalently, if

$$
\int_{\Omega}\left|f_{j}\right|^{q} d \mu \rightarrow 0
$$

whenever $f_{j} \rightarrow 0$ weakly in $b^{p}$. Note that the kernels $k_{x, p}$ converge to 0 weakly in $b^{p}$ as $x \rightarrow \partial \Omega$ for each $1<p<\infty$; see Lemma 3.10 of [4].

We also characterize vanishing $(p, q)$-Carleson measures. We first consider the case $p \leq q$.

Theorem 3.5. Let $\mu \geq 0$. Assume $1<p \leq q<\infty, s=p / q, 1 / s<t<\infty$, and $\delta, \varepsilon \in(0,1)$. Then the following conditions are equivalent:
(a) $\mu$ is a vanishing $(p, q)$-Carleson measure.
(b) $\tilde{\mu}_{t}(x) r(x)^{n(1-1 / s)} \rightarrow 0$ as $x \rightarrow \partial \Omega$.
(c) $\hat{\mu}_{\varepsilon}(x) r(x)^{n(1-1 / s)} \rightarrow 0$ as $x \rightarrow \partial \Omega$.
(d) $\hat{\mu}_{\delta}\left(a_{m}\right) r\left(a_{m}\right)^{n(1-1 / s)} \rightarrow 0$ as $m \rightarrow \infty$. 
Proof. First, suppose (a) and show (b) with $t=q$. Since $k_{x, p} \rightarrow 0$ weakly in $b^{p}$ as $x \rightarrow \partial \Omega$, it follows from (3.1) that (b) holds for $t=q$.

Next, suppose (b) and show (c). By Lemma 2.13, we have (c) for $\varepsilon<\delta_{0}$. But, an simple application of Lemma 2.2 shows that (c) holds for a given $\varepsilon$.

The implication (c) $\Rightarrow$ (d) follows from Lemma 2.2 and (2.1) as before, because $a_{m} \rightarrow$ $\partial \Omega$ as $m \rightarrow \infty$.

Now, assume (d) and show (a). Let $\left\{f_{k}\right\}$ be a sequence converging to 0 weakly in $b^{p}$. Let $M_{j}=\sup _{m \geq j} \hat{\mu}_{\delta}\left(a_{m}\right) r\left(a_{m}\right)^{n(1-1 / s)}$ for positive integers $j$. By the proof of $(\mathrm{d}) \Rightarrow$ (a) of Theorem 3.1, we have

$$
\int_{\Omega}\left|f_{k}\right|^{q} d \mu \lesssim \sum_{m<j} \int_{E_{\delta}\left(a_{m}\right)}\left|f_{k}\right|^{q} d V+M_{j}\left\|f_{k}\right\|_{p}^{q}
$$

for each $j$ and $k$. Since $f_{k} \rightarrow 0$ weakly in $b^{p}$, one can easily see that $f_{k} \rightarrow 0$ uniformly on compact subsets of $\Omega$ and $\left\{f_{k}\right\}$ is bounded in $L^{p}$-norm. Thus, fixing $j$ and taking the limit $k \rightarrow \infty$ in (3.4), we obtain

$$
\underset{k}{\limsup } \int_{\Omega}\left|f_{k}\right|^{q} d \mu \lesssim M_{j}
$$

for each $j$. Note that we have $M_{j} \rightarrow 0$ as $j \rightarrow \infty$ by assumption. Thus, taking the limit $j \rightarrow \infty$, we conclude

$$
\underset{k}{\limsup } \int_{\Omega}\left|f_{k}\right|^{q} d \mu=0 .
$$

Namely, $\mu$ is a vanishing $(p, q)$-Carleson measure, as desired.

Finally, as in the proof of Theorem 3.1, one can see that (a) implies (b) for general $t$.

The case $p>q$ is a little bit more subtle and we have the following.

THEOREM 3.6. Let $\mu \geq 0$ and assume $1<q<p<\infty$. Then the following conditions are equivalent:

(a) $\mu$ is a $(p, q)$-Carleson measure.

(b) $\mu$ is a vanishing $(p, q)$-Carleson measure.

ProOF. We only need to prove (a) $\Rightarrow$ (b). So assume $\mu$ is a $(p, q)$-Carleson measure. Take any sequence $\left\{f_{j}\right\}$ converging weakly to 0 in $b^{p}$. Then $\left\{f_{j}\right\}$ is a bounded sequence in $b^{p}$ and $f_{j} \rightarrow 0$ on each compact subset of $\Omega$. Let $K$ be any compact subset of $\Omega$ and $\mu_{K}$ be the restriction of $\mu$ to $\Omega \backslash K$. Let $s=p / q$ and fix $\varepsilon \in(0,1)$. Then, as in the proof of (c) $\Rightarrow$ (a) of Theorem 3.4, we have

$$
\int_{\Omega \backslash K}\left|f_{j}\right|^{q} d \mu=\int_{\Omega}\left|f_{j}\right|^{q} d \mu_{K} \lesssim\left\|f_{j}\right\|_{p}^{q}\left\|\hat{\mu}_{K, \varepsilon}\right\|_{s^{\prime}}
$$

for all $j$. Therefore, letting $M=\sup _{j}\left\|f_{j}\right\|_{p}^{q}<\infty$, we have

$$
\int_{\Omega}\left|f_{j}\right|^{q} d \mu \lesssim\left\{\int_{K}\left|f_{j}\right|^{p} d \mu\right\}^{q / p}+M\left\|\hat{\mu}_{K, \varepsilon}\right\|_{s^{\prime}} .
$$


Take the limit $j \rightarrow \infty$. Since $f_{j}$ converges to 0 uniformly on $K$, we have

$$
\limsup \int_{\Omega}\left|f_{j}\right|^{q} d \mu \lesssim M\left\|\hat{\mu}_{K, \varepsilon}\right\|_{s^{\prime}} .
$$

Note that $\hat{\mu}_{K, \varepsilon} \rightarrow 0$ as $K$ increases to $\Omega$. Also, we have $\left|\hat{\mu}_{K, \varepsilon}\right|^{s^{\prime}} \leq\left|\hat{\mu}_{\varepsilon}\right|^{s^{\prime}} \in L^{1}$ by Theorem 3.4. Thus, an application of the dominated convergence theorem yields

$$
\underset{j}{\limsup } \int_{\Omega}\left|f_{j}\right|^{q} d \mu=0,
$$

and therefore $\mu$ is a vanishing $(p, q)$-Carleson measure.

4. Toeplitz operators. In this section, we characterize Toeplitz operators with positive symbols in terms of Carleson measures.

By the theorems we have proved in Section 3, the notion of (vanishing) $(p, q)$-Carleson measures depend only on the ratio $p / q$. Thus, in what follows, (vanishing) $(p, q)$-Carleson measures will be simply called (vanishing) $s$-Carleson measures where $s=p / q$. For $0<$ $s<\infty$, we let $W^{s}=W^{s}(\Omega)$ denote the class of all $s$-Carleson measures. Also, we let $W_{0}^{s}=W_{0}^{s}(\Omega)$ denote the class of all vanishing $s$-Carleson measures. Note that $W^{s_{1}} \subset W^{s_{2}}$ for $0<s_{1}<s_{2}<\infty$.

The first step towards our characterization is to justify the "formal" equality

$$
\left\langle T_{\mu} f, g\right\rangle=\int_{\Omega} f \bar{g} d \mu, \quad f, g \in b^{\infty}
$$

which can be seen by formally exchanging the order of integrations after representing $T_{\mu} f$ as an integral and then applying the reproducing property. Of course, we cannot expect that such a formal argument applies to all $\mu \geq 0$. So, we will let $W^{\infty}=W^{\infty}(\Omega)$ denote the class of all $\mu \geq 0$ for which (4.1) holds. It turns out that $W^{\infty}$ contains all $W^{s}$, which justifies the notation $W^{\infty}$.

LEMMA 4.1. $\bigcup_{0<s<\infty} W^{s} \subset W^{\infty}$.

Proof. Let $\mu \in W^{s}$ for some $0<s<\infty$. We may assume $s>1$. Note that the function $y \mapsto \int_{\Omega}|R(x, y)| d x$ is subharmonic on $\Omega$. Also, by Lemma 2.6, we have

$$
\int_{\Omega}|R(x, \cdot)| d x \lesssim \Phi 1,
$$

where $\Phi$ is the operator defined in (2.3). Fix $\delta \in(0,1)$. Since $\mu \in W^{s}$, we have $\hat{\mu}_{\delta} \in L^{s^{\prime}}$ by Theorem 3.4. Also, we have $\Phi 1 \in L^{s}$ by Lemma 2.9. Thus, by Lemma 2.11, we have

$$
\int_{\Omega} \int_{\Omega}|R(x, y)| d x d \mu(y) \lesssim \int_{\Omega}(\Phi 1) \hat{\mu}_{\delta} d V<\infty .
$$


Therefore, for $f, g \in b^{\infty}$, we conclude by Fubini's theorem that

$$
\begin{aligned}
\left\langle T_{\mu} f, g\right\rangle & =\int_{\Omega} \overline{g(x)} \int_{\Omega} R(x, y) f(y) d \mu(y) d x \\
& =\int_{\Omega} f(y) \int_{\Omega} R(x, y) \overline{g(x)} d x d \mu(y) \\
& =\int_{\Omega} f(y) \overline{g(y)} d \mu(y) .
\end{aligned}
$$

We now characterize bounded (resp. compact) Toeplitz operators in terms of (resp. vanishing) $s$-Carleson measures. We first consider the case $p \leq q$.

THEOREM 4.2. Let $1<p \leq q<\infty, 1 / s=1-1 / q+1 / p$ and $\mu \geq 0$. Then the following conditions are equivalent:

(a) $T_{\mu}: b^{p} \rightarrow b^{q}$ is bounded (resp. compact).

(b) $\mu \in W^{s}$ (resp. $\left.W_{0}^{s}\right)$.

In the proof below, we will use fact that the dual of $b^{p}$ is $b^{p^{\prime}}$ for $1<p<\infty$ under the usual pairing

See Corollary 4.3 of [6].

$$
\langle f, g\rangle=\int_{\Omega} f \bar{g} d V
$$

Proof. Assume (a) and show (b). First, assume that $T_{\mu}: b^{p} \rightarrow b^{q}$ is bounded. Let $x \in \Omega$ and take $\delta=\delta_{0}$, where $\delta_{0}$ is the number provided by Lemma 2.12. Then, we have

$$
\mu\left(E_{\delta}(x)\right) \lesssim r(x)^{2 n} \int_{\Omega}|R(x, y)|^{2} d \mu(y)=r(x)^{2 n} T_{\mu}[R(x, \cdot)](x),
$$

and therefore

$$
\hat{\mu}_{\delta}(x) \lesssim r(x)^{n} T_{\mu}[R(x, \cdot)](x) \approx r(x)^{n\left(1-1 / p^{\prime}\right)} T_{\mu} k_{x, p}(x) .
$$

On the other hand, we have by Proposition 8.1 of [2]

$$
\left|T_{\mu} k_{x, p}(x)\right| \lesssim r(x)^{-n / q}\left\|T_{\mu} k_{x, p}\right\|_{q} .
$$

Combining these estimates, we have

$$
\hat{\mu}_{\delta}(x) r(x)^{n(1-1 / s)} \lesssim\left\|T_{\mu} k_{x, p}\right\|_{q} .
$$

Now, since $\left\|k_{x, p}\right\|_{p}=1$, we see that

$$
\hat{\mu}_{\delta}(x) r(x)^{n(1-1 / s)} \lesssim\left\|T_{\mu}\right\|,
$$

where $\left\|T_{\mu}\right\|$ denote the operator norm of $T_{\mu}: b^{p} \rightarrow b^{q}$. This is true for all $x \in \Omega$ and the constants abbreviated above are independent of $x$. Hence, $\mu \in W^{s}$ by Theorem 3.1.

Recall that $k_{x, p} \rightarrow 0$ weakly in $b^{p}$ as $x \rightarrow \partial \Omega$. Hence, if $T_{\mu}: b^{p} \rightarrow b^{q}$ is compact, then we have by (4.2)

$$
\hat{\mu}_{\delta}(x) r(x)^{n(1-1 / s)} \lesssim\left\|T_{\mu} k_{x, p}\right\|_{q} \rightarrow 0
$$

as $x \rightarrow \partial \Omega$. Hence, $\mu \in W_{0}^{s}$ by Theorem 3.5 . 
Now, assume (b) and show (a). First, assume $\mu \in W^{s}$. Let $f, g \in b^{\infty}$. Since $1 / s=$ $1 / q^{\prime}+1 / p$, we note that $p / s$ is the conjugate exponent of $q^{\prime} / s$. By Lemma 4.1 , we have

$$
\begin{aligned}
\left|\left\langle T_{\mu} f, g\right\rangle\right| & =\left|\int_{\Omega} f \bar{g} d \mu\right| \\
& \leq\left\{\int_{\Omega}|f|^{p / s} d \mu\right\}^{s / p}\left\{\int_{\Omega}|g|^{q^{\prime} / s} d \mu\right\}^{s / q^{\prime}} \\
& \lesssim\|f\|_{p}\|g\|_{q^{\prime}} .
\end{aligned}
$$

The last inequality holds by our assumption $\mu \in W^{s}$. Now, a duality argument shows the boundedness of $T_{\mu}: b^{p} \rightarrow b^{q}$, because $b^{\infty}$ is dense in $b^{p}$.

Next, assume $\mu \in W_{0}^{s}$. Let $\left\{f_{j}\right\}$ be a sequence of functions such that $f_{j} \rightarrow 0$ weakly in $b^{p}$. Then we have

$$
\int_{\Omega}\left|f_{j}\right|^{p / s} d \mu \rightarrow 0
$$

Hence, by (4.3) and a duality argument, we obtain

$$
\left\|T_{\mu} f_{j}\right\|_{q} \lesssim\left\{\int_{\Omega}\left|f_{j}\right|^{p / s} d \mu\right\}^{s / p} \rightarrow 0 .
$$

Therefore, $T_{\mu}: b^{p} \rightarrow b^{q}$ is compact.

Now, we turn to the case $q<p$. In this case we will prove that bounded Toeplitz operators are all compact.

LEMMA 4.3. Let $s>1$ and $\mu \in W^{\infty}$. Then the following conditions are equivalent:

(a) $T_{\mu}: b^{p} \rightarrow b^{q}$ is bounded whenever $1<q<p<\infty$ and $1 / s=1-1 / q+1 / p$.

(b) $T_{\mu}: b^{2 s} \rightarrow b^{2 s /(2 s-1)}$ is bounded.

(c) $\mu \in W^{s}$.

Proof. The implication (a) $\Rightarrow$ (b) is trivial.

Assume (b) and show (c). Let $s_{1}=2 s /(2 s-1)>1$. Then $s_{1}^{\prime}=2 s$. Since $\mu \in W^{\infty}$ by assumption, it follows from (4.1) that

$$
\int_{\Omega}|f|^{2} d \mu=\left|\left\langle T_{\mu} f, f\right\rangle\right| \leq\left\|T_{\mu}\right\|\|f\|_{2 s}^{2}
$$

for any $f \in b^{\infty}$, where $\left\|T_{\mu}\right\|$ denotes the operator norm of $T_{\mu}: b^{2 s} \rightarrow b^{2 s /(2 s-1)}$. Hence, $\mu \in W^{s}$. (a).

Finally, as in the proof of (b) $\Rightarrow$ (a) of Theorem 4.2, we have the implication (c) $\Rightarrow$

THEOREM 4.4. Let $s>1$ and $\mu \in W^{\infty}$. Then the following conditions are equivalent:

(a) $T_{\mu}: b^{p} \rightarrow b^{q}$ is compact whenever $1<q<p<\infty$ and $1 / s=1-1 / q+1 / p$.

(b) $T_{\mu}: b^{p} \rightarrow b^{q}$ is bounded whenever $1<q<p<\infty$ and $1 / s=1-1 / q+1 / p$.

(c) $T_{\mu}: b^{2 s} \rightarrow b^{2 s /(2 s-1)}$ is compact.

(d) $T_{\mu}: b^{2 s} \rightarrow b^{2 s /(2 s-1)}$ is bounded. 
(e) $\mu \in W_{0}^{s}$.

(f) $\mu \in W^{s}$.

Proof. By Lemma 4.3 we have the equivalences $(\mathrm{b}) \Leftrightarrow(\mathrm{d}) \Leftrightarrow(\mathrm{f})$. By Theorem 3.6, we have the equivalence (e) $\Leftrightarrow$ (f). The implications (a) $\Rightarrow$ (c) $\Rightarrow$ (d) are trivial. Also, we have (e) $\Rightarrow$ (a), as in the proof of (b) $\Rightarrow$ (a) of Theorem 4.2.

We now close the paper with the following remark.

REMARK. The hypothesis $\mu \in W^{\infty}$ in Lemma 4.3 is used in the proof of the implication $(b) \Rightarrow(c)$. In case the domain has enough symmetry like a ball, it turns out that Condition (b) itself implies $\mu \in W^{\infty}$. Note that Condition (b) implies

$$
T_{\mu}\left(b^{\infty}\right) \subset b^{1} .
$$

This weaker condition actually yields $\mu \in W^{\infty}$ on the ball, as in the following.

Let $\Omega=B$ be the unit ball in $\mathbf{R}^{n}$. The advantage we will utilize is the fact that the kernel function for $B$ has the additional symmetry

$$
R(t x, y)=R(x, t y), \quad x, y \in B, \quad 0 \leq t \leq 1,
$$

which can be easily seen from the explicit formula given in Theorem 8.13 of [2]. Now, let $\mu \geq 0$ and assume that (4.5) holds. Let $t \in(0,1)$ and write $\chi_{t}$ for the characteristic function of $t B=\{t x ; x \in B\}$. Note that $R(\cdot, \cdot)$ is bounded on $t B \times B$ by Lemma 2.6. Thus, for $f, g \in b^{\infty}$, we have by Fubini's theorem

$$
\begin{aligned}
\left\langle T_{\mu} f, g \chi_{t}\right\rangle & =\int_{t B} \overline{g(x)} \int_{B} f(y) R(x, y) d \mu(y) d x \\
& =\int_{B} f(y) \int_{t B} \overline{g(x)} R(x, y) d x d \mu(y) \\
& =t^{n} \int_{B} f(y) \int_{B} \overline{g(t x)} R(t x, y) d x d \mu(y) \\
& =t^{n} \int_{B} f(y) \int_{B} \overline{g(t x)} R(x, t y) d x d \mu(y) \\
& =t^{n} \int_{B} f(y) \overline{g\left(t^{2} y\right)} d \mu(y) .
\end{aligned}
$$

Thus, taking the limit $t \rightarrow 1$, we conclude $\mu \in W^{\infty}$ by the dominated convergence theorem.

So, in the case of the ball, the hypothesis $\mu \in W^{\infty}$ in Lemma 4.3, and hence in Theorem 4.4 , is redundant.

\section{REFERENCES}

[ 1 ] K. R. Attele, Analytic multipliers of Bergman spaces, Michigan Math. J. 30 (1984), 307-319.

[2] S. Axler, P. Bourdon And W. Ramey, Harmonic function theory, Springer-Verlag, New York, 1992.

[ 3 ] B. R. Choe, H. Koo And H. Yi, Positive Toeplitz operators between the harmonic Bergman spaces, Potential Anal. 17 (2002), 307-335. 
[ 4 ] B. R. Choe, Y. J. LeE And K. NA, Toeplitz operators on harmonic Bergman spaces, Nagoya Math. J. 174 (2004).

[ 5 ] J. Cima And W. Wogen, A Carleson measure theorem for the Bergman space on the ball, J. Operator Theory 7 (1982), 157-165.

[6] H. KAng AND H. Koo, Estimates of the harmonic Bergman kernel on smooth domains, J. Funct. Anal. 185 (2001), 220-239.

[ 7 ] D. LuECKING, Embedding theorem for spaces of analytic functions via Khinchine's inequality, Michigan Math. J. 40 (1993), 333-358.

[ 8 ] D. Luecking, Multipliers of Bergman spaces into Lebesgue spaces, Proc. Edinburgh Math. Soc. 29 (1986), $125-131$.

[ 9 ] D. Luecking, Trace ideal criteria for Toeplitz operators, J. Funct. Anal. 73 (1987), 345-368.

[10] J. Miao, Reproducing kernels for harmonic Bergman spaces of the unit ball, Monatsh. Math. 125 (1998), 25-35.

[11] J. MiAo, Toeplitz operators on harmonic Bergman spaces, Integral Equations Operator Theory 27 (1997), 426-438.

[12] O. L. OLEINIK, Embedding theorems for weighted classed of harmonic and analytic functions, J. Soviet Math. 9 (1978), 228-243.

[13] K. ZHU, Positive Toeplitz operators on weighted Bergman spaces of bounded symmetric domains, J. Operator Theory 20 (1988), 329-357.

DEPARTMENT OF MATHEMATICS

KOREA UNIVERSITY

SEOUL 136-701

KOREA

E-mail address: choebr@math.korea.ac.kr

DePARTMENT OF MATHEMATICS

KOREA UNIVERSITY

SEOUL 136-701

KOREA

E-mail address: nakyunguk@korea.ac.kr
DEPARTMENT OF MATHEMATICS

MOKPO NATIONAL UNIVERSITY

CHONNAM 534-729

KOREA

E-mail address: yjlee@mokpo.ac.kr 